\title{
Fisiopatologia dell'iperparatiroidismo secondario
}

\section{F. Malberti}

Divisione di Nefrologia e Dialisi, Istituti Ospitalieri, Cremona

Negli ultimi anni sono stati fatti considerevoli progressi nella conoscenza dei meccanismi fisiopatologici dell'iperparatiroidismo secondario (IPTS).

Ricerche di base e studi su modelli animali hanno portato a una migliore conoscenza delle funzioni biologiche del recettore per il calcio (CaSR), del recettore per la vitamina $\mathrm{D}$ (VDR), dell'ormone fosfaturico "fibroblastic growth factor 23" (FGF23), dei meccanismi di regolazione della sintesi della 1,25-diidrossivitamina D o calcitriolo (1-25D) e dell'attività del sistema enzimatico CYP27BI-CYP24A1 (o $1 \alpha$ idrossilasi - 24 idrossilasi). Nonostante le recenti acquisizioni vi è attualmente controversia in letteratura su quale sia il primo movens nello sviluppo dell'IPTS. Accanto a una visione "classica", che ha goduto di molti consensi negli ultimi anni, e che vede il deficit di 1-25D quale fattore scatenante l'IPTS, ha ripreso consenso una visione "fosforo-centrica", sostenuta da diversi autori già agli inizi degli anni Settanta (1), in cui la ritenzione di fosforo come conseguenza della riduzione della massa nefronica sarebbe il primo stimolo allo sviluppo dell'IPTS.

\section{Ruolo del deficit di Calcitriolo (1-25D)}

L'IPTS si sviluppa precocemente nel corso dell'insufficienza renale (IR) come meccanismo adattativo per mantenere l'omeostasi calcio-fosforica. Un aumento di livelli paratormone (PTH) si evidenzia in alcuni pazienti già nello stadio 2 ed è presente in molti pazienti nello stadio 3 (2-4). L'aumento dei livelli di PTH è preceduto dalla riduzione dei livelli plasmatici di 1-25D, mentre l'aumento della fosforemia si osserva in una fase più avanzata dell'IR (2-4). Secondo alcuni autori $(5,6)$, l'IR sarebbe una condizione caratterizzata già in fase precoce da deficit di vitamina $\mathrm{D}$, principalmente dovuta a una ridotta capacità renale di convertire il calcidiolo, o $25 \mathrm{OH}$-vitamina $\mathrm{D}$ (25D), nella vitamina $\mathrm{D}$ attiva, cioè la $1-25 \mathrm{D}$, per una ridotta attività dell'enzima $1 \alpha$ idrossilasi. Un altro fattore che contribuisce alla riduzione di 125D nell'IR è la ridotta disponibilità di substrato per la $1 \alpha$ idrossilasi dovuto a minore riassorbimento tubulare di $25 \mathrm{D}$ come conseguenza della riduzione del filtrato glomerulare $(7,8)$. La $25 \mathrm{D}$ per essere idrossilata deve infatti venire riassorbita dalle cellule tubulari prossimali a opera del recettore megalina $(7,8)$. I ridotti livelli plasmatici di $25 \mathrm{D}$ sono un altro fattore limitante la sintesi di 1-25D. Diversi studi hanno evidenziato la presenza di una carenza di vitamina D (ridotti livelli plasmatici di 25D) nell'IR $(4,9)$. La riduzione dei livelli di 1-25D favorisce lo sviluppo di IPTS con due meccanismi, uno diretto (diminuita soppressione della sintesi di PTH), l'altro indiretto (riduzione dell'assorbimento intestinale di calcio e con seguente riduzione della calcemia). Nella Figura 1 sono schematizzati i meccanismi fisiopatologici dell'IPTS in uno scenario che vede il deficit di 1-25D come fattore scatenante. L'aumento dei livelli di PTH consente di mantenere la calcemia e la fosforemia nel range di normalità fino alle fasi più avanzate dell'IR quando i meccanismi di compenso diventano inefficaci. Lo stimolo protratto nel tempo porta ad ipertrofia ed iperplasia paratiroidea. 


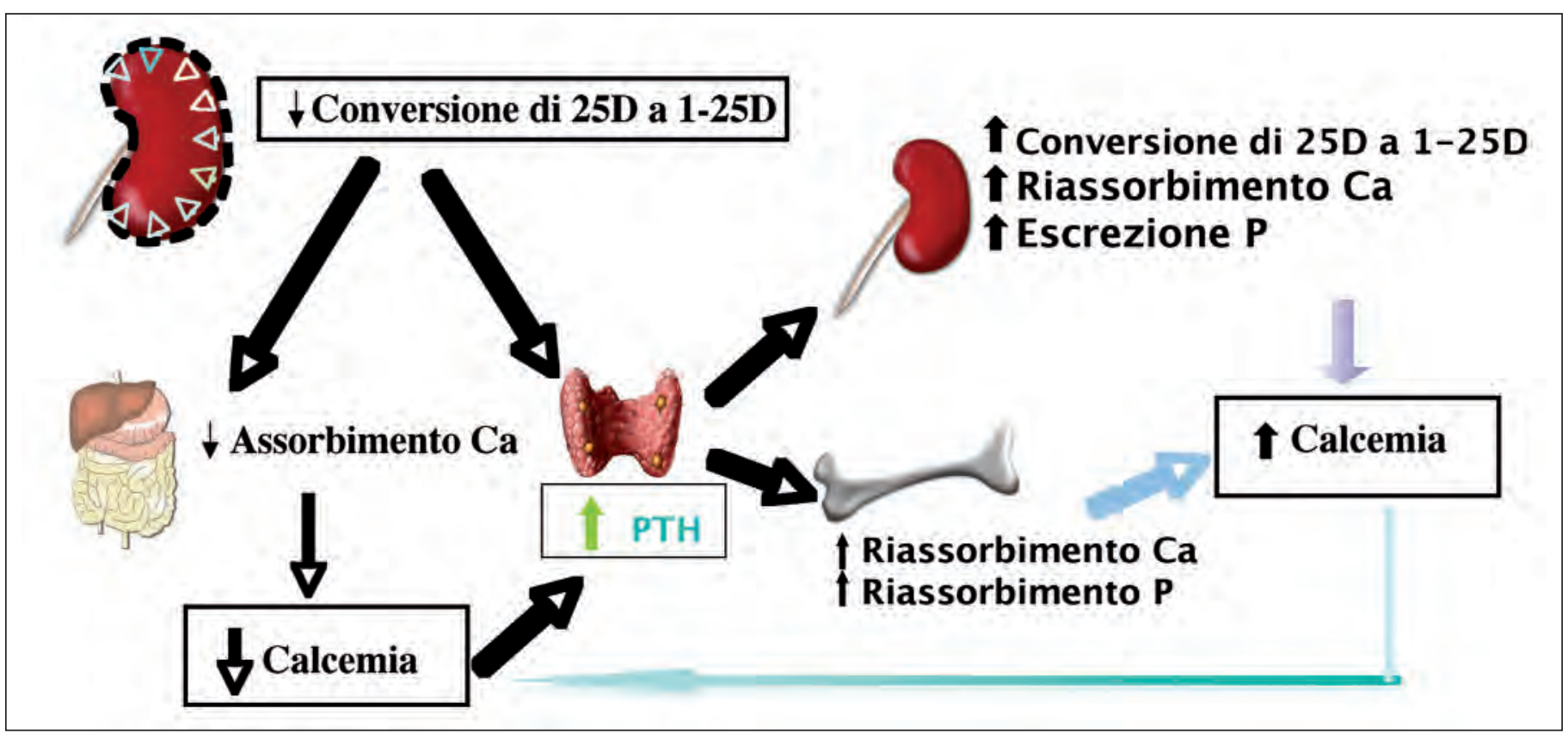

Fig. 1 - La ridotta sintesi di 1-25D determina un aumento del PTH con un meccanismo diretto (ridotta soppressione della sintesi di PTH) e indiretto (ridotto assorbimento intestinale di calcio ed ipocalcemia). L'aumento del PTH stimola il rene ad aumentare la sintesi di 1-25D, e il riassorbimento tubulare di calcio e determina un aumento del riassorbimento osseo (con conseguente efflusso di calcio e fosforo dall'osso). Il risultato netto è il mantenimento di normali livelli di calcemia. La fosforemia rimane normale perché l'efflusso di fosforo dall'osso è compensato dall'aumento dell'escrezione renale di fosforo.

\section{Ruolo del Fosforo}

Diverse osservazioni mettono in discussione la visione tradizionale in cui l'IPTS sarebbe la diretta conseguenza del deficit di 1-25D. Innanzitutto, con il ridursi della funzione renale vi è una minore capacità del rene a eliminare il fosforo e la riduzione dell'apporto dietetico di fosforo di per sé è in grado di prevenire lo sviluppo di IPTS nei modelli animali (10). Secondo, il deficit di vitamina D si associa generalmente a ipofosforemia più che a iperfosforemia. Terzo, la funzione principale dell'asse PTH-vitamina D è la prevenzione dell'ipocalcemia e non dell'iperfosforemia. L'azione fosfaturica del PTH ha come scopo principale quello di evitare la ritenzione di fosforo che accompagna l'assorbimento intestinale di calcio e l'efflusso di fosforo dall'osso che si accompagna al riassorbimento osseo di calcio.

La recente scoperta dell'FGF23, quale ormone che "contro-regola" la 1-25D, prevenendone gli effetti negativi, quale l'iperfosforemia, in caso di ipersecrezione o intossicazione da vitamina D (11), ha consentito di delucidare alcuni aspetti patogenetici dell'IPTS. Il FGF23 è una proteina costituita da 251 aminoacidi con un peso molecolare di 26.000 Da. È prodotta dagli osteociti e viene degradata in 2 frammenti, il C-terminale (aa 180-251) e l'N-terminale (aa 1-24). A livello renale il FGF23 riduce l'attività del cotrasporto sodio-fosfato di tipo IIa (NaPi2a) e modula l'attività della 1alfa-idrossilasi e della 24-idrossilasi. Sebbene sia il PTH sia il FGF23 siano fosfaturici, essi hanno azione opposta sugli enzimi $1 \alpha$ idrossilasi e 24 idrossilasi. Il PTH stimola la sintesi di 1-25D aumentando l'attività della $1 \alpha$ idrossilasi e riducendo l'attività della 24 idrossilasi, che degrada la 1-25D in forma inattiva. Esattamente opposta è l'azione del FGF23 sulle due idrossilasi (12). La precoce elevazione del FGF23 nel corso dell'IR (13) contribuisce alla riduzione dei livelli circolanti di 1-25D e spiega la scarsa efficacia dei supplementi di vitamina D (ergocalciferolo, colecalciferolo) nell'aumentare i livelli plasmatici di 1-25D (14). Infatti, il FGF23 è un predittore indipendente di bassi livelli di 1-25D dopo aggiustamento per i livelli circolanti di 25D (14). Pertanto, l'asse FGF23-osso-rene potrebbe essere l'effettore della "trade-off" ipotesi sul ruolo del fosforo nella patogenesi dell'IPTS secondario. Come schematizzato nella Figura 2, la ridotta escrezione di fosforo è il primo stimolo a una cascata di eventi nei quali la soppressione della produzione rena- 


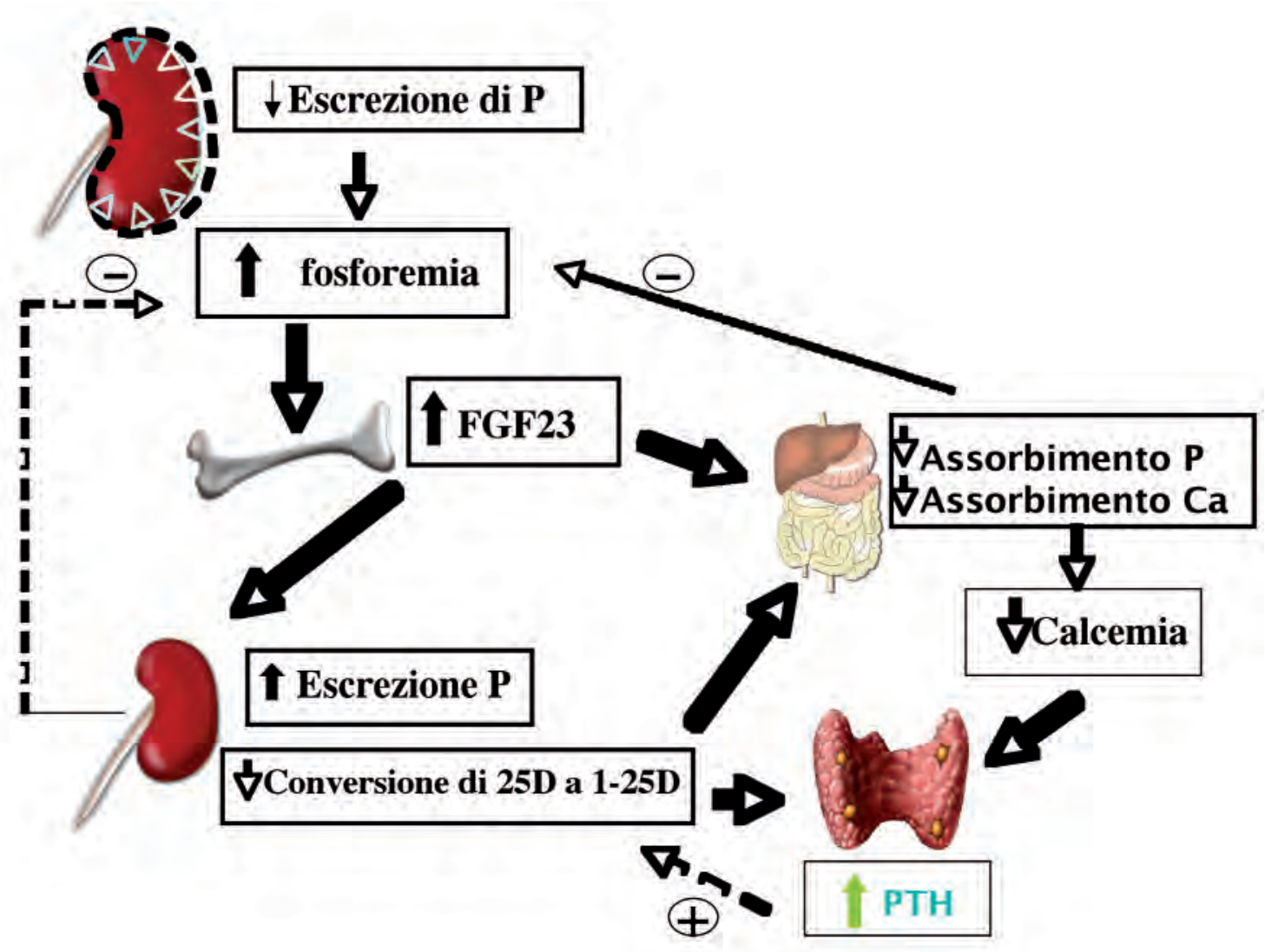

Fig. 2 - La riduzione dell'escrezione renale di fosforo conseguente alla riduzione della massa nefronica stimola la produzione ossea di FGF23. Il FGF23 inibisce il riassorbimento tubulare renale di fosforo e riduce la sintesi renale di 1-25D. La fosforemia è mantenuta a livelli normali dall'aumentata escrezione renale e dal ridotto riassorbimento intestinale conseguente alla riduzione della 1-25D. La riduzione dei livelli plasmatici di 1-25D determina riduzione della calcemia per ridotto assorbimento intestinale con conseguente stimolo alla secrezione del PTH, come nella interpretazione patogenetica tradizionale. Nell'interpretazione "fosforocentrica" il primo movens che porta allo sviluppo dell'IPTS è la ritenzione di fosforo con aumento dei livelli plasmatici di FGF23, mentre l'abbassamento dei livelli plasmatici di 1-25D sarebbe un fenomeno più tardivo.

le di 1-25D a opera del FGF23 è la risposta adattativa necessaria a limitare l'assorbimento intestinale di fosforo, in modo tale da mantenerne neutro il bilancio corporeo. Il FGF23 contribuirebbe ai bassi livelli di $1-25 \mathrm{D}$, riducendo il PTH (12), che ha un'azione di stimolo sull'attività della $1 \alpha$ idrossilasi. L'incremento del FGF23 è un fenomeno precoce nel corso dell'IR e sarebbe, secondo alcuni, la principale causa di riduzione dei livelli plasmatici di $1-25 \mathrm{D}$, mentre l'incremento del PTH sarebbe un fenomeno tardivo conseguente alla riduzione dei livelli di 1-25D. Nella Figura 3 sono schematizzatii meccanismi che limitano la capacità del rene a mantenere normali livelli di 1-25D nel corso dell'IR.

A livello cellulare, l'effetto del FGF23 è mediato dal klotho, che è una glucosidasi presente sulla membrana cellulare in grado di legare il recettore per gli FGF trasformandolo in un recettore specifico per il FGF23 (15). Dato che l'espressione del RNA messaggero per il klotho è ridotta nei pazienti con IR, gli elevati livelli circolanti di FGF23 nella IR avanzata si spiegano anche con una resistenza d'organo al FGF23 (16).

\section{Nuovi aspetti}

Uno studio recente (17) ha suggerito che vi possono essere ipotesi alternative alla ridotta capacità di escrezione renale del fosforo e ai ridotti livelli plasmatici di 1-25D atte a spiegare lo sviluppo IPTS nelle fasi iniziali 


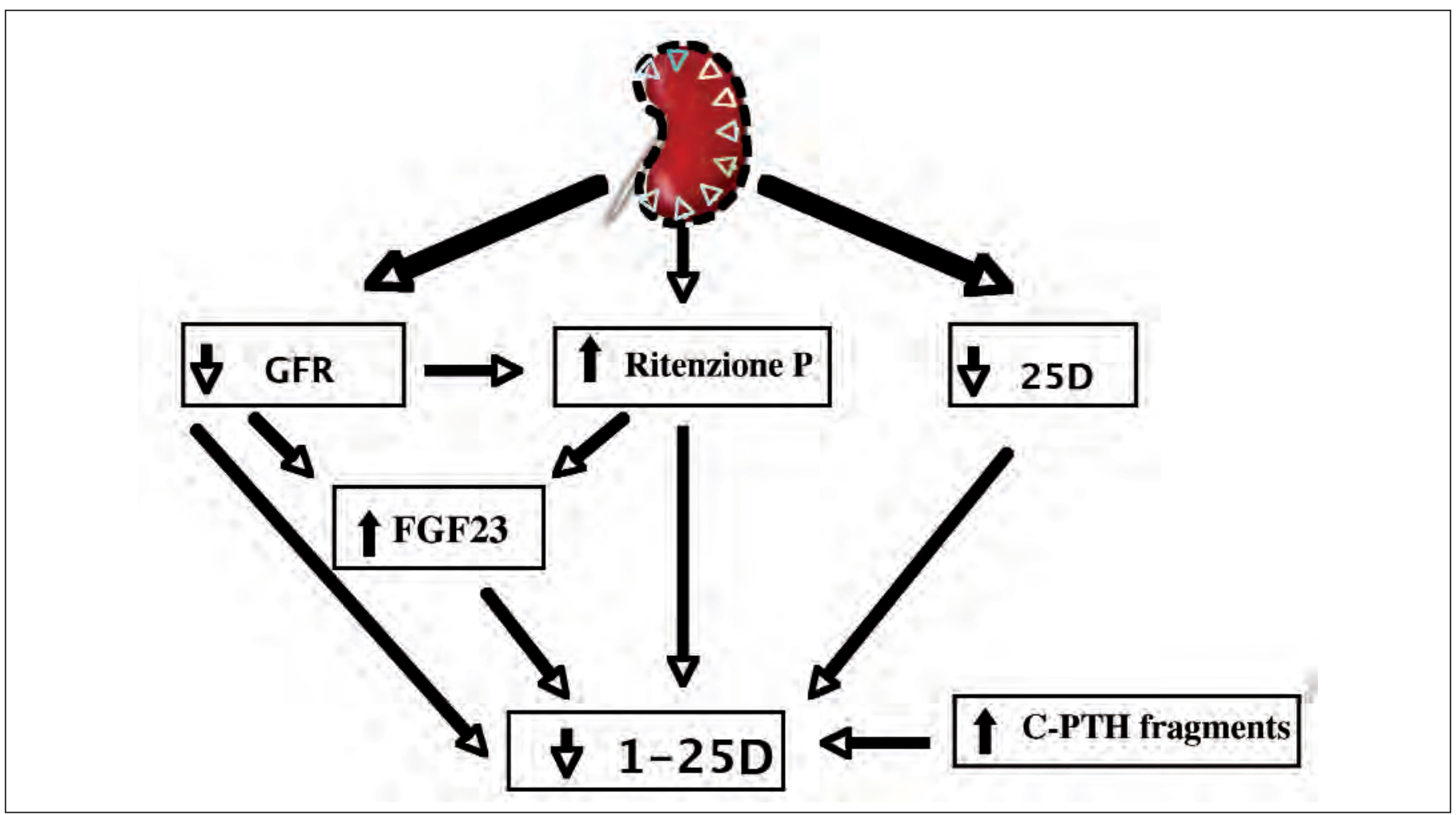

Fig. 3 - Meccanismi coinvolti nel limitare la capacità del rene di mantenere i livelli plasmatici di 1-25D. La riduzione della massa nefronica riduce la capacità di idrossilare la 25D in 1-25D. La riduzione del substrato (deficit di 25D), frequente nell'IR, è un altro fattore che limita la sintesi di 1-25D. L'incremento del FGF23, precoce nel corso dell'IR, sarebbe, secondo alcuni, la principale causa di riduzione dei livelli plasmatici di 125D. Un altro fattore coinvolto nel ridurre l'attività della 1-alfa-idrossilasi sarebbe l'aumento in circolo dei frammenti N-terminali e C-terminali del PTH (29).

dell'IR. Allo scopo di valutare se nel periodo post-prandiale vi fossero stimoli alla produzione di PTH e FGF23, Isakova et al (17), hanno confrontato l'effetto di pasti a differente contenuto di calcio e fosforo in 13 pazienti con IR (GFR medio $41 \mathrm{~mL} / \mathrm{min}$ ) e in 21 controlli sani. In condizioni basali, i pazienti con IR avevano, rispetto ai normali, più elevati livelli basali di FGF23 ed escrezione frazionaria di fosforo e minore escrezione frazionaria di calcio, mentre non erano diversi i livelli plasmatici di calcio, fosforo e PTH. Lo studio ha evidenziato che nelle 4 ore post-prandiali l'escrezione urinaria di fosforo aumentava in entrambi i gruppi senza variazioni della fosforemia e del FGF23. L'escrezione urinaria di calcio aumentava in entrambi i gruppi, ma si accompagnava a riduzione della calcemia ed aumento del PTH solo nei pazienti con IR. Pertanto, secondo gli autori, un'inappropriata calciuria postprandiale con ipocalcemia potrebbe essere un altro possibile meccanismo patogenetico dell'IPTS nei pazienti con IR.

\section{Ruolo dei recettori per il calcio (CaSR) e per la vitamina $D$ (VDR)}

Il CaSR regola la secrezione di PTH, la trascrizione del gene del PTH e la proliferazione delle cellule paratiroidee (18). Nei topi knock-out per il CaSR si hanno ipercalcemia, elevati livelli di PTH e iperplasia paratiroidea nonostante elevati livelli plasmatici di 1-25D (19). Nei topi l'ablazione dei VDR causa iperplasia paratiroidea e iperparatiroidismo, ma la normalizzazione della calcemia è sufficiente a correggere l'iperplasia paratiroidea e gli elevati livelli di PTH (20). Il fatto che la 1-25D sia inefficace nel sopprimere la produzione di PTH in assenza dei CaSR e che la normalizzazione della calcemia sia sufficiente a normalizzare la funzione paratiroidea in assenza di VDR, indica che i CaSR hanno un ruolo dominante nella regolazione della funzione paratiroidea $(21,22)$. A livello paratiroideo, la principale funzione dei VDR è la soppressione della trascrizione del gene del PTH; inoltre, l'attivazione dei VDR eserci- 


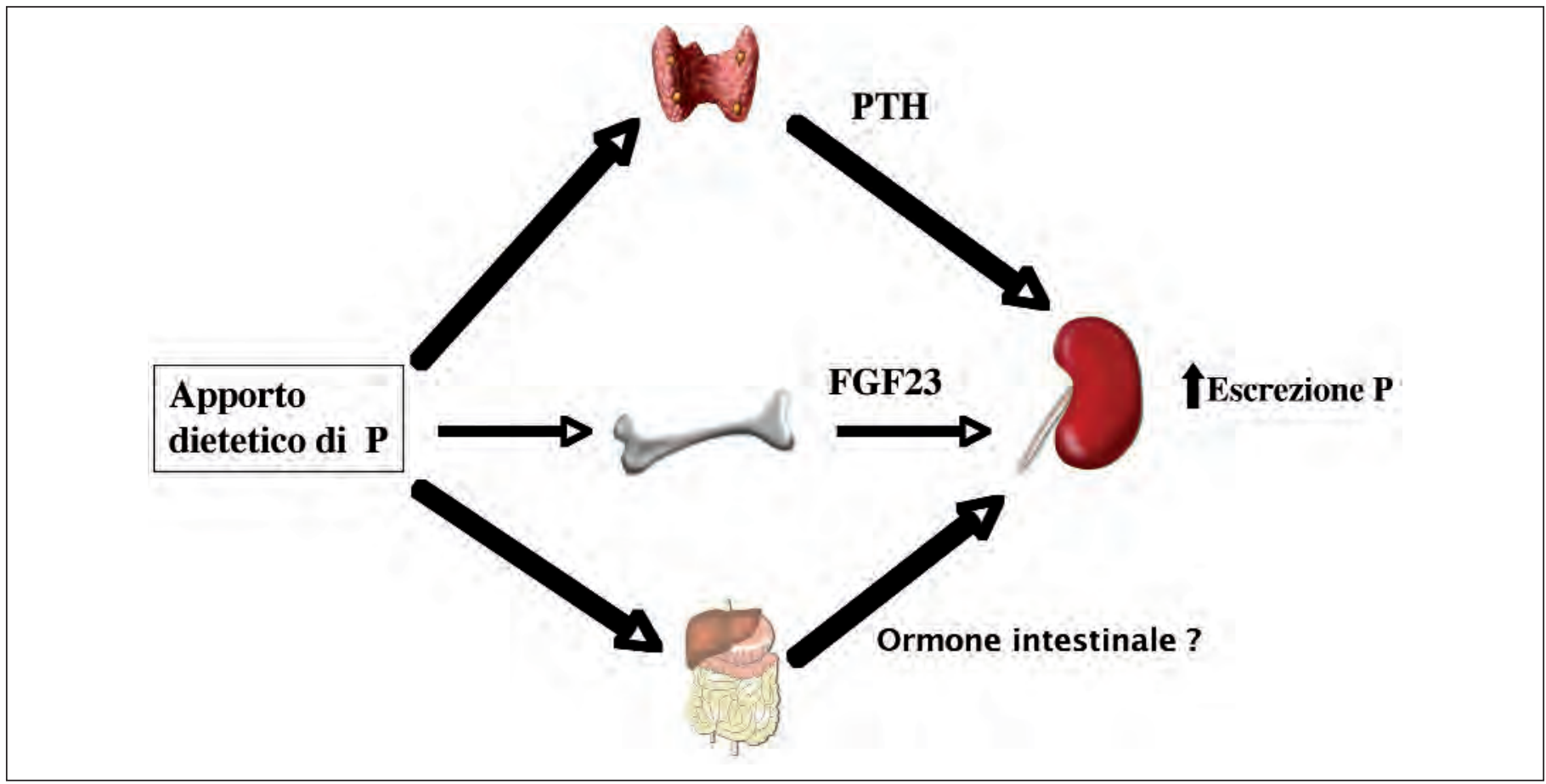

Fig. 4 - È schematizzata la regolazione del bilancio fosforico. L'apporto dietetico di fosforo stimola l'escrezione urinaria di fosforo (verosimilmente ad opera di un ormone non ancora identificato), aumenta il PTH e il FGF23, che a loro volta inducono fosfaturia.

ta un effetto soppressivo indiretto sulle paratiroidi attraverso l'aumento del riassorbimento intestinale di calcio e l'aumento della calcemia, che inibisce le paratiroidi attraverso i CaSR. Inoltre l'attivazione dei VDR determina una upregulation dei CaSR (23). Per contro, i livelli di calcemia regolano l'espressione dei VDR (24). Nell'IPTS severo si ha una downregulation dei VDR e CaSR $(25,26)$, che rende le paratiroidi resistenti alla terapia con metaboliti attivi della vitamina D (27).

\section{Conclusioni}

Negli ultimi anni è emerso il ruolo chiave del FGF23 nella regolazione dell'omeostasi del fosforo. Inoltre, la documentazione che l'apporto dietetico di fosforo determina fosfaturia con un meccanismo d'azione rapido (minuti) indipendentemente da variazione dei livelli plasmatici di calcio, fosforo, PTH e FGF23 (28), ha supportato l'ipotesi dell'esistenza di un asse entero-renale di regolazione omeostatica. Nella Figura 4 è schematizzata la regolazione del bilancio fosforico. L'apporto dietetico di fosforo stimola l'escrezione urinaria di fosforo (verosimilmente a opera di un ormone non ancora identificato), aumenta il PTH e il FGF23, che a loro volta inducono fosfaturia. Il FGF23, riducendo la produzione renale di 1-25D, previene un ulteriore assorbimento intestinale di fosforo, e contribuisce al ristabilimento dell'omeostasi fosforica. Questo asse è alterato già nelle fasi iniziali dell'IR; tuttavia, i potenziali effetti negativi e le conseguenze dell'elevazione del FGF23 e della precoce riduzione dei livelli plasmatici di 125D devono essere ancora del tutto chiarite. Rimane ancora da chiarire il meccanismo attraverso il quale il fosforo regola la produzione di FGF23 e il meccanismo con cui le cellule percepiscono le variazioni della fosforemia, non essendo ancora stato identificato un recettore cellulare per il fosforo. Infine, l'emergere di questo asse "osso-renale" avrà implicanze terapeutiche, che potrebbe modificare il corrente approccio nell'IR iniziale. Sebbene l'uso della vitamina $\mathrm{D}$ rimanga il caposaldo terapeutico nell'IR iniziale, l'osservazione che l'apporto dietetico di fosforo stimola la produzione di FGF23, che, a sua volta riduce la sintesi di $1-25 \mathrm{D}$, perpetuandone il deficit, suggerisce che la restrizione dell'apporto dietetico di fosforo deve essere prescritto precocemente nell'IR cronica. 
Indirizzo degli Autori:

Fabio Malberti, MD

Divisione di Nefrologia e Dialisi

Istituti Ospitalieri

26100 Cremona

f.malberti@ospedale.cremona.it

\section{Bibliografia}

1. Slatopolsky E, Caglar S, Pennell JP, Toggart J, Canterbury J, Reiss E, Bricker NS. On the pathogenesis of hyperparathyroidism in chronic experimental insufficiency in the dog. J Clin Invest 1971; 50: 497-9.

2. Martinez I, Saracho R, Montenegro J, Llach F. The importance of dietary calcium and phosphorous in the secondary hyperparathyroidism of patients with early renal failure. Am J Kidney Dis1997; 29: 496502.

3. De Boer IH, Gorodetskaya I, Young B, et al. The severity of secondary hyperparathyroidism in chronic renal insufficiency is GFR-Dependent, racedependent, and associated with cardiovascular disease. J Am Soc Nephrol 2002; 13: 2762-9.

4. Levin A, Bakris GL, Molitch M. Prevalence of abnormal serum vitamin D, PTH, calcium, and phosphorus in patients with chronic kidney disease: Results of the study to evaluate early kidney disease. Kidney Int 2007; 71: 31-8.

5. Al-Badr W, Martin KJ. Vitamin D and kidney disease. Clin J Am Soc Nephrol 2008: 3: 1555-60.

6. Brown AJ, Slatopolsky E. Drug insight: vitamin D analogs in the treatment of secondary hyperparathyroidism in patients with chronic kidney disease. Nat Clin Pract Endocrnol Metab 2007; 3: 134-44.

7. Nykjaer A, Fyfe JC, Kozyraki R, et al. Cubilin dysfunction causes abnormal metabolism of the steroid hormone 25(OH) vitamin D(3). Proc Natl Acad Sci USA 2001; 98: 13895-900.

8. Hilpert J, Wogensen L, Thykjaer T, et al. Expression profiling confirms the role of endocytic receptor megalin in renal vitamin D3 metabolism. Kidney Int 2002; 62: 1672-81.

9. Ravani P, Malberti F, Tripepi G, et al. Vitamin D levels and patients outcome in chronic kidney disease. Kidney Int 2009; 75: 88-95.

10. Slatopolsky E, Role of phosphorous in the pathogenesis of secondary hyperparathyroidism. Am J Kidney Dis 2001; 37 (Suppl): S54-57.

11. Liu S, Tang W, Zhou J, et al. Fibroblastic growth fac- tor 23 is a counter-regolatory phosphaturic hormone for vitamin D. J Am Soc Nephrol 2006; 17: 1305-15.

12. Krajisnik T, Bjorklund P, Marsell R, et al. Fibroblastic growth factor 23 regulates parathyroid hormone and 1 alpha-hydroxylase expression in cultured bovine cells. J Endocrinology 2007; 195: 125-31.

13. Larsson T. Circulating concentration of FGF23 increases as renal function declines in patients with chronic kidney disease, but does not change in response to variation in phosphate intake in healthy volunteers. Kidney Int 2003; 64: 2272-79.

14. Gutierrez O, Isakova T, Rhee E, et al. Fibroblastic growth factor 23 mitigates hyperphosphatemia but accentuates calcitriol deficiency in chronic kidney disease. J Am Soc Nephrol 2005; 16: 2205-15.

15. Urakawa I. Klotho converts canonical FGF receptor into a specific receptor for FGF23. Nature 2006; 444: 770-4.

16. Koh N. Severely reduced production of klotho in human chronic renal failure kidney. Biochem Biophys Res Commun 2001; 280: 1015-20.

17. Isakova T, Gutierrez O, Shah A, Castaldo L, Holmes J, Lee H, Wolf M. Postprandial mineral metabolism and secondary hyperparathyroidism in early CKD. J Am Soc Nephrol 2008; 19: 615-23.

18. Brown EM. Clinical lesons from the calcium-sensing receptor. Nat Clin Pract Endocrinol Metab 2007; 3: 122-33.

19. Ho C. A mouse model of human familial hypocalciuric hypercalcemia and neonatal severe hyperparathyroidism. Nat Genet 1995; 11: 389-94.

20. Li YC. Normalization of mineral ion homeostasis by dietary means prevents hyperparathyroidism, rickets, and osteomalacia, but not alopecia in vitamin d receptor-ablated mice. Endocrinology 1998; 139: 4391-6.

21. Kos $\mathrm{CH}$. The calcium-sensing receptor is required for normal calcium homeostasis independent of parathyroid hormone. J Clin Invest 2003; 111: 1021-8.

22. Goodman WG. Calcimimetics: a remedy for all problems of excess parathyroid hormone activity in 
chronic kidney disease. Curr Opin Nephrol Hypertens 2005 ; 14 : 355-60.

23. Brown AJ. Rat calcium-sensing receptor is regulated by vitamin D but not by calcium. Am J Physiol 1996; 270: F454-60.

24. Maiti A, Beckman MJ. Extracellular calcium is a direct effector of VDR levels in proximal tubule epitelial cells that counter-balances effects of PTH on renal vitamin $\mathrm{D}$ metabolism. J Steroid Biochem Mol Biol 2007; 103: 504-8.

25. Kifor O, Moore FD, Wang P, White M. Reduced immuno-staining for extracellular $\mathrm{Ca} 2+-$ sensing receptor in primary or secondary uremic hyperpara- thyroidism. J Clin Endocrinol Metab 1996; 81: 1598-606.

26. Fukuda N, Tanaka H, Tominaga Y, Fukagawa M, Kurokawa K. Decreased 1,25 dihydroxyvitamin D receptor density is associated with a more severe form of parathyroid hyperplasia in chronic uremic patients. J Clin Invest 1993; 92: 1436-43.

27. Malberti F, Corradi B, Cosci P, Calliada F, Marcelli D, Imbasciati E. Long-term effects of intravenous calcitriol therapy on the control of secondary hyperparathyroidism. Am J Kidney Dis 1996; 28: 704-12.

28. Berndt T, Thomas LF, Craig Ta, et al. Evidence for a signaling axis by which intestinal phosphate rapidly modulates renal phosphate reabsorption. Proc Nat Acad Sci USA 2007; 104: 11085-90.

29. Usatii M, Rousseau L, Demers C, et al. Parathyroid hormone fragments inhibit active hormone and hypocalcemia-induced 1,25(OH)2D synthesis. Kidney Int 2007; 72: 1330-5. 